\title{
Strategies for Activating Historic District: Micro-reform Implement in Qiulin District, Harbin
}

\author{
Binyu $\mathrm{Lei}^{1, *}$, Qi Gao ${ }^{1}$, and Yanyao $\mathrm{Cui}^{1}$ \\ ${ }^{1}$ Urban Planning and Design Institute of Shenzhen, Shenzhen, China
}

\begin{abstract}
This paper explores urban strategies that address existing problems in Qiulin District, which is famous as a historic district with plenty of cultural memories in Harbin, China. In the paper, it firstly introduces the definition of historic district and made a brief review of academic literatures about space activation. It then takes Qiulin District in Harbin as a case to further discuss the current dilemma. In terms of land use, Qiulin District is primarily commercial and residential that increases the difficulty of activating this area. In accordance with demonstrated issues: 1) heritage buildings with limited value capture, 2) diverse business types challenged with urgent upgrading, 3) multiple high-quality resources utilized in weak synergy, it proposes three main strategies to solve problems. With introducing the concept of micro-reform, the strategies: 1) activate heritage buildings to recapture historic value, 2) extend outdoor space and upgrade commercial structure to enhance characteristic streets, 3) design for fun life to build inclusive network, compressively explore a number of possibilities to reflect the current situation and achieve visionary planning. Finally, this paper draws discussion about the significance of space activation for historic districts towards environmental sustainability and human beings.
\end{abstract}

\section{Introduction}

Historic district, as part of cultural heritage, significantly contributes to urban context. Basically, historic district is considered as an important carrier of urban cultural environment [1]. According to the State Ministry of Housing and Urban-Rural Development in China (2015), it lists a total of 30 historic and cultural streets and districts regarding cultural value and economic benefits [2]. However, during the process of urban development, it has become growing concern about activation of historic districts. On the one hand, traditional spatial patterns have increasingly decayed, which are hard to adapt to modern built environment. On the other hand, the shortage of land resource and the mass construction of real estate inevitably pose a threat against urban heritage preservation [3].

Researches on activating historic districts are mainly concluded in two perspectives: renewal and protection [4]. Mumford (1961) makes a critique of urban construction in macro-scale ignoring city's vitality [5]. Jacobs (1961) also demonstrates the significance of historic areas during the process of urban regeneration movement [6]. It literally emphasizes preservation of urban heritage in terms of its integrity. However, since the twenty-first century, historic district conservation is further challenged by more complicated conditions [7]. The concept of organic renewal is raised to promote microscale guide planning towards historic areas [8]. It addresses every historic district as a whole with integrating numerous cultural elements and modern parts as well. Also, the activation of historic districts not only remains in the discussion of government or other stakeholders, but also associates with public participation [9]. In 2015, United Nations Educational, Scientific and Cultural Organization (UNESCO) proposes "Operational Guidelines for the Implementation of the World Heritage Convention", adapting Historic Urban Landscape (HUL) as an innovative approach to value urban heritage [10]. HUL approach is dynamic in response to changes over time including built environment, human behaviors and historic heritage itself. The present of HUL approach extends the scope of historic conservation which aims to include it as the media reconnecting culture and society [11]. It largely relieves contradiction between urban renewal and heritage preservation, and also suggests new perspective to activate historic streets and districts. In recent decade, studies on activation of urban historic districts more focus on social cohesion and activation tactics in micro-scale, rather than static perspective on protection [12]. Space activation nowadays pays more attention to revitalize historic district without destroying its original features. Moreover, it attaches importance to public engagement with consideration of numerous demands to achieve an inclusive and sustainable neighborhood.

\section{Case study: Qiulin District}

Qiulin District is a typical example of heritage conservation in Harbin, located in the heart of the city boasting a history spanning over more than a hundred

\footnotetext{
* Corresponding author: leiby@upr.cn
} 
years (Figure 1). The development of Qiulin District entirely reflects the history of culture and society. In 1900, the Churin Foreign Trading Company established by the Russians and became the most popular shopping mall. With the rapid growth of Churin Company, it conducted diverse business across countries and attracts a large number of tourists from Europe and Asia. Subsequently, more types of buildings were built by foreigners for residence and commerce, that forming a cluster of foreign-culture buildings. The company and other constructions have sustained a history of more than 120 years while nowadays they have no functions as before only reserving the heritage building. A hundred years flashing by, Qiulin District, as the origin of the city, inherits the initial layout and preserves a number of delicate historical buildings, delivering its unique urban texture. At present, Qiulin District has become an essential identify of Harbin through one hundred year of construction and preservation.

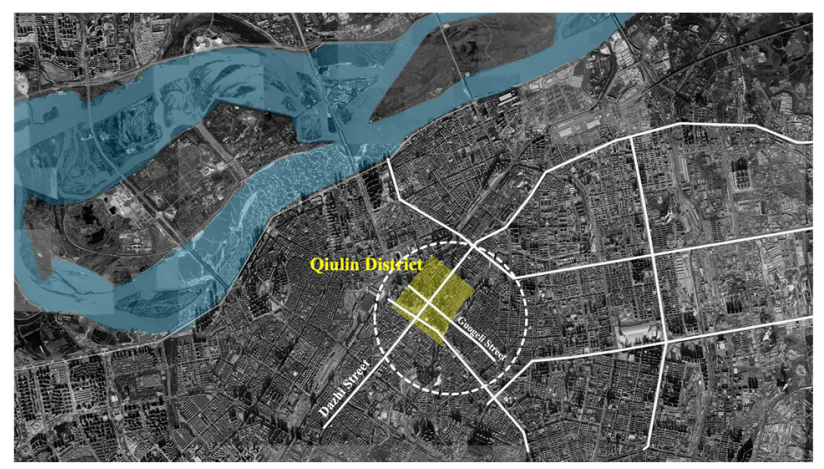

Fig. 1. The location of Qiulin District.

Qiulin District occupies an area of 1.43 in the center of Harbin. The land use in this area is mainly commercial and residential, as well as a small proportion of public space for services like education and administration (Table 1).

Table 1. The provision of land use in Qiulin District.

\begin{tabular}{|l|c|c|}
\hline Categories & Area $/ m^{2}$ & Proportion/\% \\
\hline Residential & 1181202 & 43.68 \\
\hline Commercial & 1261066 & 46.64 \\
\hline Public space & 253059 & 9.36 \\
\hline Residential & 1181202 & 43.68 \\
\hline
\end{tabular}

In general, today's Qiulin District is highly mixed with diverse urban functions. Historic buildings are gradually transformed into museums, schools, malls and other functions while a number of new dwellings are built for residence (Figure 2). The Museum of Heilongjiang Province, the Youth Palace of Harbin, and the Activity Center, together with other public infrastructures in Qiulin District, manifest the potential to rebuild a cultural circle in the contemporary era. In terms of heritage buildings,
Qiulin District gathers a cluster of Modern European Style buildings, represented by the consulates and consulate generals of several countries, including Russia, Britain, Italy, France, and Japan. They concentrically distribute in the west and east of Qiulin District, mainly along the Dazhi Street and Guogeli Street. Such distinct architectural heritage reflects the cultural communication between China and the Western countries. Although the original purposes have passed, the cultural buildings themselves still have a profound impact on the development of Harbin and play a significant role in the new era.

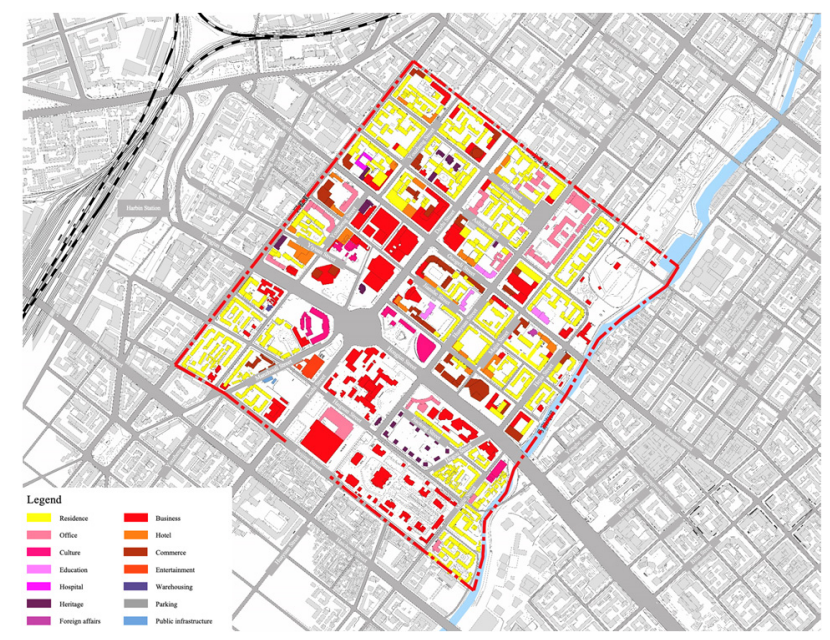

Fig. 2. The architectural nature of Qiulin District.

However, with the rapid development, the heritage buildings have lost their original charm. The historic value has not been fully captured. A part of historical buildings is faced with total vacancy or insufficient utilization, some of which are poorly maintained due to illegal extensions or reconstructions. The building clusters scatter across this area with poor connectivity, disrupting the intrinsic ambience. In terms of industrial structure, a large proportion of the business types in Qiulin District stagnate in the traditional formats, in want of transition and progression to the latest forms, that fail the synchronous development of new era (Figure 3). As consumption upgrades and innovative economy grows, the young generation has shifted their predilections for various exhibitions, brand shops, fashion events. The old business model is hard to continue with a lack of attractiveness to satisfy people's demand at the present. The functions of multiple heritage buildings in Qiulin District, such as commercial shopping centers, and cultural facilities, lack inner linkage that poorly maintain the integrality as a whole. Some cultural facilities and historical buildings are relatively enclosed, sealing the built-in yards and other open space off from interaction with the public. Pedestrian access to public space in this area is blocked by vehicle pathways, resulting in fragmentary spaces and diminishing dynamics. In consideration of numerous problems existed, Qiulin District urgently needs adaptation to the up-to-date demands, deeply mining its own features and value, so as to augment its regional competitiveness and appeal to more diverse groups. 

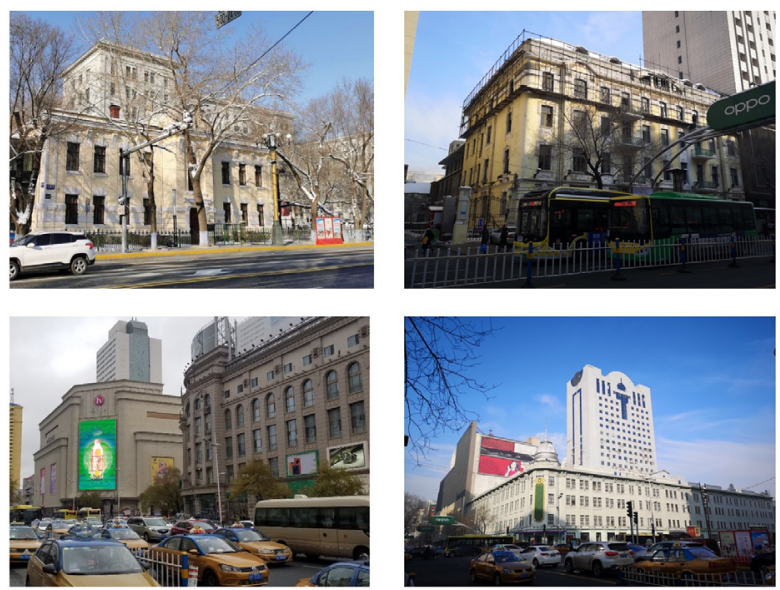

Fig. 3. The status quo of Qiulin District.

\section{Strategies for space activation}

In 2016, "Measures for Urban Renewal of Guangzhou" is introduced by the Guangzhou Municipal Government to guide and manage the activation of historic districts, which nowadays widely applied to practice in China. It firstly introduces "micro-reform", which is implemented through partial demolition, preservation or renovation on the premise of maintaining the basic construction patterns [13]. Literally, micro-reform is a flexible way to capture historic value and adapt heritage for new era development. In spatial pattern, it addresses the architectural features with continuing traditional culture. In addition, microreform implementation of historic districts considerably emphasizes environmental sustainability, as primarily keeping heritage as an essential part of natural resources. [14]. It considers the regeneration of heritage that provides physical inputs into social services and experience. Meanwhile, the strategy of micro-reform activation takes indigenous peoples into serious consideration. It largely manages the cost of construction without threatening the security of original property right [15]. Micro-reform implement provides a comprehensive method to activate historic districts regarding historic value and multiple subjects in the process of urban renewal. According to status quo of Qiulin District, it develops three main strategies to draw micro-reform, including cultural core regeneration, historic streets preservation and life enhancement (Figure 4).
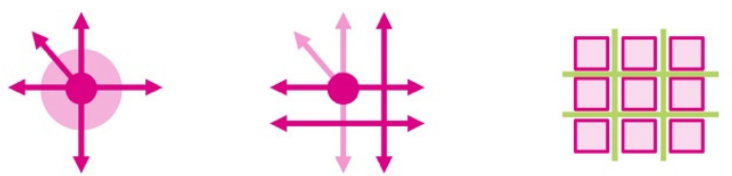

Fig. 4. Three main strategies (cultural core, historic streets and life network).

\subsection{Strategy 1: Build Sky Walk to recapture value in the culture core}

The culture core in Qiulin District is surrounded by Youzheng Street, Jianshe Street, Huayuan Street and Haiguan Street. There are 16 heritage buildings in this area, which include 2 Embassies and Consulates buildings, a history museum, the children's palace and 7 public squares. Since the lag of construction from the last century, the culture core nowadays presents a chaotic spatial pattern losing attraction in the new era. The primary thing for the existing area is to recapture its value, in order to awaken the vitality and promote attractive atmosphere. Through building the vertical floor-connection system (Sky Walk), it connects historical buildings and integrate cultural gardens (Figure 5). In general, it bridges scattered elements to increase connectivity and provide more access to explore historic value in this area.

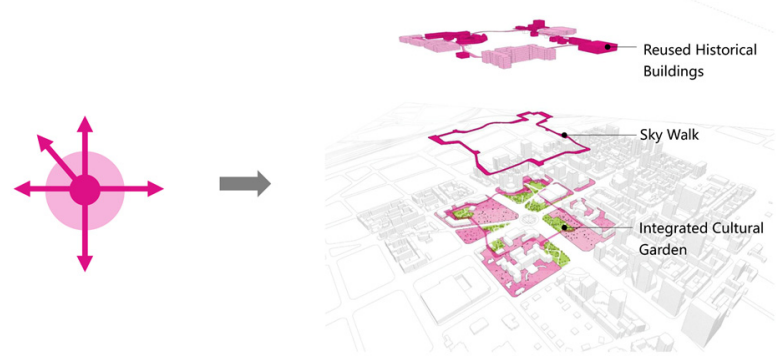

Fig. 5. Build the culture core.

In terms of representative buildings in Qiulin District, there are two tactics to reuse them and stimulate their vitality. Firstly, the replacement of original functions conforms to current urban development. It is widely received as a contemporary way towards environmental sustainability, which largely maintains the historic elements and introduces modern functions, like innovation and creative design, to promote cultural integration. For example, it encourages the conservation of historical features and transforms the inner function to exhibition or experience, like the Creative Museum and the VR Experience Center (Figure 6).

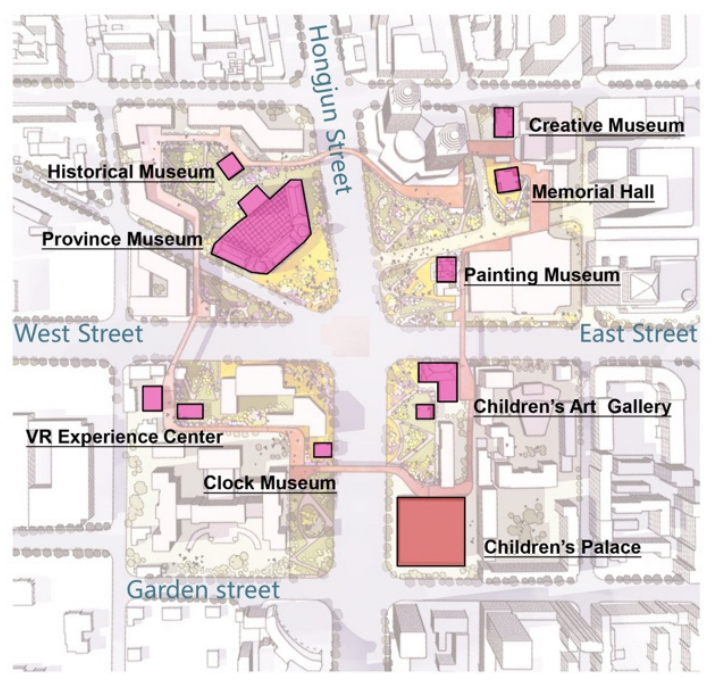

Fig. 6. Reused heritage buildings.

The other tactic is to extend existing heritage buildings to satisfy more demands in the new era and support 
tourism development as well, like the Historical Museum and the Province Museum. It attaches public services to heritage buildings where people have more chances to learn about the precious history [16]. In order to improve access to reused buildings, the Sky Walk system with corridors ensures the comfortable wandering of pedestrians in the historic district to freely enter heritage buildings [17]. It also links scattered small squares and plenty of green space in this area, to basically provide sufficient activity space regarding walking consistency (Figure 7).

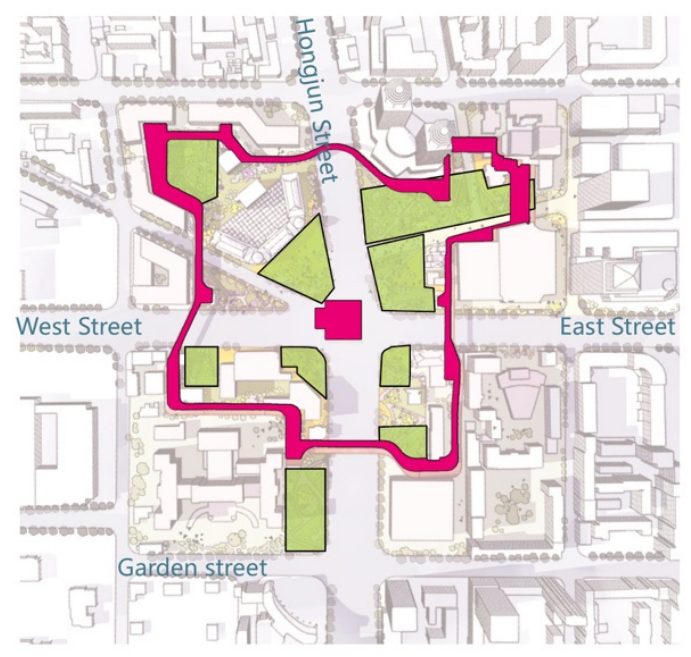

Fig. 7. The Sky Walk.

\subsection{Strategy 2: Enhance characteristic streets to satisfy contemporary development}

Dazhi Street, Huayuan Street and Guogeli Street are arterial streets in Qiulin District, presenting unique landscape and cultural environment in this area (Figure 8). Basically, the three streets combine numerous historic elements including heritage buildings and public infrastructures, which entirely reflect the flux of social history. However, the existing problems limit the sustainable development. The traffic-oriented development largely weakens the cultural ambience of commercial pedestrian streets. The heritage buildings scattered throughout the streets fail to demonstrate their historical value. Thus, the enhancement of historic characteristics is a considerable method to address the competitiveness on account of current situation.
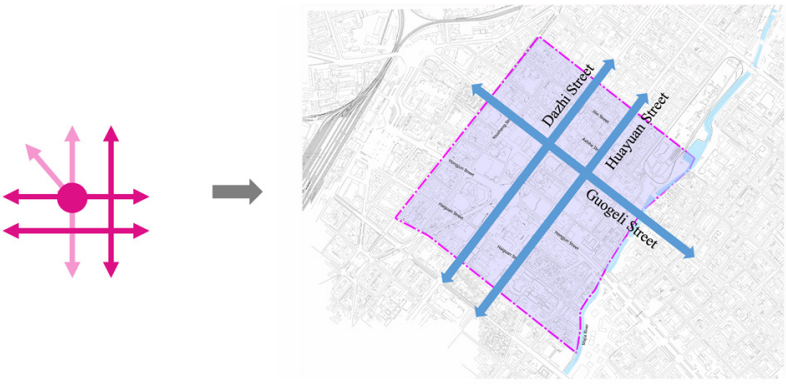

Fig. 8. Enhance characteristic streets.
It takes the Guogeli Street as an example. Without changing the red line, road separation will fully release more public space through separating the current streets. It alleviates traffic jams against the heavy use of private cars, and ensures the safety of pedestrians as well [18]. The released commercial space is open to the public designed as a catalyst to drive the revival of heritage buildings, potentially engaging people in walking along the street and pursuing leisure activities [19]. Also, the outdoor space provides a panoramic view to completely enjoy historic landscape. For instance, depending on diverse historic features, cultural events, like Russian Food Festival, will be regularly held in the extension commercial space to improve the vitality of Guogeli Street. In accordance with emerging consumer demands, the industrial structure needs to introduce new industries, such as fashion shopping, snacks and bars, in order to complete upgrading. The vertical business model is regarded as an effective way to upgrade the industrial formats for the sake of full mix-use in order to well maintain the historical façade along the Guogeli Street. It insets office functions in dwellings and adds first-floor business to meet daily demands, like bookshop or café (Figure 9). It sufficiently builds diverse industrial forms to improve functions to enhance multi-purpose development, and preserves the historic atmosphere along the arterial street as well.

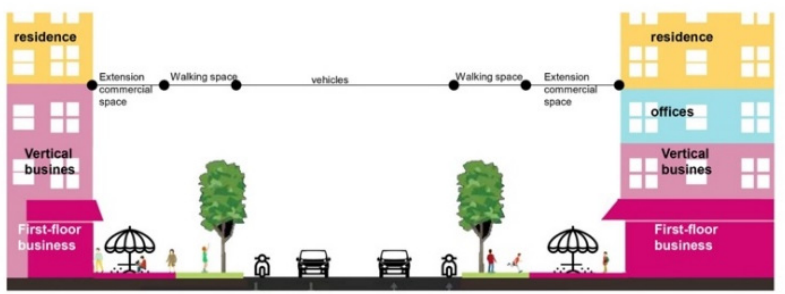

Fig. 9. Conduct road separation.

\subsection{Strategy 3: Design for fun life}

On the basis of existing open small blocks and courtyardstyle space in Qiulin District, a more interesting and highquality urban living neighborhood will be built (Figure 10). It sets up small facilities in the block to satisfy various activities and makes full use of inefficient corner space to build pocket parks. The entire network is systematically designed to improve the quality of life with activation of the historic district. People are attracted to engage in this area and enjoy city life with traditional ambience. The future growth of population in next decades will stimulate various demands for public facilities. Outdoor space is essential for people where they have opportunities to interact with others and enjoy physical activities [20]. It requires to provide adequate facilities along the street, such as benches, where people feel free to seat and have recreation. Scattered corner space is designed for pocket parks, that intend to provide more public space for people to resume social activities and explore historic value. Pocket parks make use of roadside green belts and other negative space in line with local conditions to refine landscape. For example, around the culture core, there 
will be built numerous pocket parks providing sufficient space for leisure activities, where people are able to experience the essence of history as well as natural resources with aesthetic design. Green space along the street and within small blocks are addressed as an important component in the city where people have open access to contact with nature through an ecological environment [21].

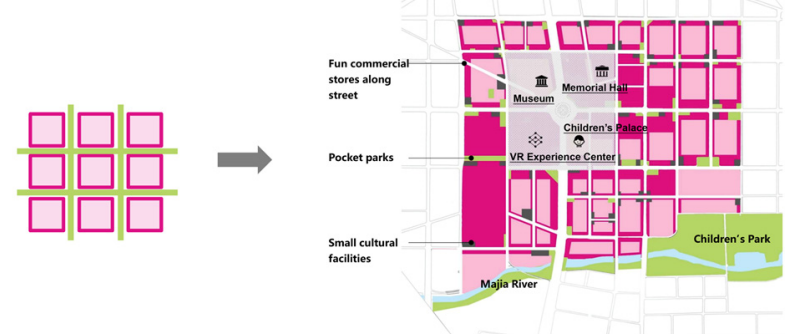

Fig. 10. Design for fun life.

\section{Discussion}

Micro-reform implement is widely considered as an effective method to address the activation of historic district that preserves the cultural value and adapts multiple functions for new development. With taking Qiulin District as an example, it should be pointed out that the origin of Qiulin District is prestigious regarding heritage buildings in collaboration with cultural elements and aesthetic sense. The activation of this special area is not a simple matter of regeneration to keep abreast of times. More importantly, it has to explore a number of possibilities to awaken the vitality towards the historic value and future demands. In consideration of land use in Qiulin District, it highly mixes residential and commercial space as well as some public services. There are three main strategies proposed to revitalize this area in order to achieve a historic and inclusive neighborhood. Some strategies emphasize the combination of historic elements and new functions, like shopping and other relaxation, to stimulate further tourism development. Other proposed actions aim to explore more possibilities for current and future demands of people living in this area. The microreform to activate Qiulin District comprehensively involves historic value into every aspect of physical buildings and casual experience. It seeks not only to solve the existing problems led by the lag of development, but also to enhance its characteristics and eventually build better life network.

\section{References}

1. B. S. Yeoh, \& S. Huang, Cities 13(6), 411-422 (1996)

2. T. Liu, R. J. Butler, C. Zhang, J. Archit. Conserv. 25(3), 228-251 (2019)

3. R. M. Leichenko, N. E. Coulson, D. Listokin, Urban Stud. 38(11), 1973-1987 (2001)

4. L. Dai, B. Xu, B.Wu, Area 49(1), 94-105 (2017)
5. L. Mumford, The city in history: Its origins, its transformations, and its prospect (1961)

6. J. Jacobs, The death and life of great American cities (1961)

7. B. Zhai, M. K. Ng, Cities 35, 14-25 (2013)

8. H. Kou, J. Zhou, J. Chen, S. Zhang, Sustainability 10(12), 4645 (2018)

9. K. Winson-Geideman, D. Jourdan, S. Gao, J. Real Estate Res. 33(1), 25-47 (2011)

10. K. Taylor, Landsc. Res. 41(4), 471-480. (2016)

11. F. Bandarin, R. Van Oers (Eds.), Reconnecting the city: the historic urban landscape approach and the future of urban heritage (2014)

12. F. Zeng, Z. Shen, Int. J. Environ. Res. Public Health 17(12), 4367 (2020).

13. Z. Xiaoyang, W. Shifu, F. Yan, South Architecture, 5, 15 (2017)

14. S. Wang, Y. Hu, H. He, G. Wang Sustainability 9(10), 1847 (2017)

15. A. Mozaffari, T. Jones (Eds.), Heritage Movements in Asia: Cultural Heritage Activism, Politics, and Identity (2019)

16. M. K. Smith, Issues in cultural tourism studies (2009).

17. M. Johansson, C. Sternudd, M. Kärrholm, J Urban Des (Abingdon) 21(2), 256-275 (2016)

18. Y. Liao, A. Shibata, K. Ishii, M. J. Koohsari, S. Inoue, K. Oka, J. Transp. Health 11, 73-79 (2018)

19. G. Ferilli, P. L. Sacco, G. Tavano Blessi, S. Forbici, Eur. Plan. Stud. 25(2), 241-258 (2017)

20. J. Wilks, Int. Res. Geogr. Environ. Educ. 19(1), 2538 (2010)

21. K. Shriver, Transp. Res. Record 1578(1), 64-75 (1997) 